\title{
Chemistry reduction using machine learning trained from non-premixed micro-mixing modeling: Application to DNS of a syngas turbulent oxy-flame with side-wall effects
}

\author{
Kaidi Wan ${ }^{\mathrm{a}}$, Camille Barnaud ${ }^{\mathrm{a}}$, Luc Vervisch ${ }^{\mathrm{a}, *}$, Pascale Domingo $^{\mathrm{a}}$ \\ ${ }^{a}$ CORIA - CNRS, Normandie Université, INSA de Rouen, 76801 Saint-Etienne-du-Rouvray, France
}

\begin{abstract}
A chemistry reduction approach based on machine learning is proposed and applied to direct numerical simulation (DNS) of a turbulent non-premixed syngas oxy-flame interacting with a cooled wall. The training and the subsequent application of artificial neural networks (ANNs) rely on the processing of 'thermochemical vectors' composed of species mass fractions and temperature (ANN input), to predict the corresponding chemical sources (ANN output). The training of the ANN is performed aside from any flow simulation, using a turbulent non-adiabatic non-premixed micro-mixing based canonical problem with a reference detailed chemistry. Heat-loss effects are thus included in the ANN training. The performance of the ANN chemistry is then tested aposteriori in a two-dimensional DNS against the detailed mechanism and a reduced mechanism specifically developed for the operating conditions considered. Then, three-dimensional DNS are performed either with the ANN or the reduced chemistry for additional a-posteriori tests. The ANN reduced chemistry achieves good agreement with the Arrhenius-based detailed and reduced mechanisms, while being in terms of CPU cost 25 times faster than the detailed mechanism and 3 times faster than the reduced mechanism when coupled with DNS. The major potential of the method lies both in its data driven character and in the handling of the stiff chemical sources. The former allows for easy implementation in the context of automated generation of case-specific reduced chemistry. The latter avoids the Arrhenius rates calculation and also the direct integration of stiff chemistry, both leading to a significant CPU time reduction.
\end{abstract}

Keywords: Artificial neural network, Chemistry reduction, Direct numerical simulation, Syngas 


\section{Introduction}

Accounting for the complexity of chemistry in numerical simulation of flames has been the subject of numerous studies since the 80's. Reduced and skeleton mechanisms were first discussed from the fundamentals of combustion chemistry, sometimes coupled with more or less advanced sensitivity analyses (see [1-5] and references therein). Meanwhile, the progress in the understanding of chemistry and transport phenomena has driven the development of detailed and reliable chemistry mechanisms for fuel oxidation, which could now contain up to hundreds of species and thousands of reactions [6]. To avoid dealing with too many degrees of freedom, which would need to be solved together with the flow dynamics, these chemical mechanisms are usually downsized before their introduction in computational fluid dynamics software. Thereby, more or less automated reduction techniques of these reference detailed chemical mechanisms have been discussed in the literature. These reduction approaches may combine fuel lumping, time-scale and principal component analysis or a direct numerical treatment of the chemical signals from graph analysis or genetic algorithms, approaches which may also be associated with systematic tabulation techniques [7-23] (not exhaustive list).

Considering a detailed chemical mechanism involving a number $N_{S}$ of species interacting according to $N_{R}$ elementary reactions, the reduction procedure consists of expressing the chemical rates of a number $N_{S}^{r}<N_{S}$ of species, so that the thermochemical information relevant for the problem under consideration is preserved. Robust and well-established methods exist to rank species according to their relative importance in the prediction of thermochemical properties, which should be accurately captured by the reduced scheme [11, 15, 16, 20]. This allows for determining the minimum set of $N_{S}^{r}$ species to be transported with the flow to preserve a given level of accuracy. Once this first step completed, in a second step, the burning rates are usually expressed from a reduced set of $N_{R}^{r}<N_{R}$ elementary reactions, chosen from the detailed scheme.

A different approach is attempted in this work for the second step, i.e., after the important species have been determined. With this novel strategy, there is no reduced mechanism produced per se, but instead a specific machine learning procedure is conducted to determine, from the

\footnotetext{
${ }^{*}$ Corresponding author

Email address: vervisch@coria.fr (Luc Vervisch)
} 
detailed chemical scheme, how to express the burning rates of the reduced number of species transported with the flow. The input of the neural networks is thus the thermochemical reduced vector and the output is either the vector of reaction rates or the new thermochemical vector for a given time increment, depending on the most convenient coupling procedure with the flow solver.

Such combination of the benefits of mechanism reduction and high-dimensional chemistry tabulation approaches is not fully new and has already been achieved by employing artificial neural networks (ANNs). Indeed, ANNs have received extensive attention in tabulation of combustion chemistry, since their first usage back in the 90's [24, 25], up to recent studies demonstrating that modern ANN architectures offer good predictions with great savings in CPU time and memory requirements [26-28]. Data driven approaches have also been coupled with principal component analysis for developing closure models in turbulent combustion using experimental multi-scalar measurements [29] and for building digital-twins to progress towards furnace control [30]. Along similar lines, convolutional neural networks $(\mathrm{CNN})$, based on image-like treatment of the thermochemical fields, were shown useful to tackle the modeling of turbulent flames [31-33] and their control [34].

Recently, chemistry reduction based on CNN was discussed with a training of the CNN performed from the targeted flow simulation with detailed chemistry [35]. However, for machine learning to be fully efficient and of practical interest, the training should be achieved on a canonical problem, as generic as possible, and ideally aside from the targeted flow simulation, as it was envisioned in [26, 36]. To this end, training from a non-premixed turbulent stochastic micromixing problem is discussed in this paper. This canonical problem was previously introduced in the context of automated chemistry reduction [19], with a simple pairwise micro-mixing closure. Instead, the Euclidean minimum spanning tree mixing modeling [37] is here chosen to secure a better accurate detailed chemistry response in mixture fraction space. Non-adiabatic effects are also accounted for through added enthalpy losses.

The operating conditions retained for the study are those of a syngas non-premixed oxy-flame interacting with a cooled wall. This configuration covers a wide range of combustion and turbulence conditions in terms of equivalence ratio, progress of reaction and enthalpy including thermal and viscous boundary layers effects, making it a relatively generic test case. The syngas fuel 
Table 1: Operating conditions of the syngas oxy-flame (IGAR ADEME project).

\begin{tabular}{lll}
\hline & Upper inlet & Lower inlet \\
\hline Bulk velocity [m/s] & 50 & 100 \\
Temperature [K] & 1223 & 298 \\
Mass fraction [-] & & \\
$\mathrm{H}_{2}$ & 0.0085 & 0 \\
$\mathrm{CO}$ & 0.7852 & 0 \\
$\mathrm{CO}_{2}$ & 0.0514 & 0 \\
$\mathrm{~N}_{2}$ & 0.1549 & 0 \\
$\mathrm{O}_{2}$ & 0 & 1.0 \\
\hline
\end{tabular}

composition is representative of a $\mathrm{CO}_{2}$ free blast-furnace exhaust flux, as found in modern recycling combustion processes. With the most important species determined, the response of the stochastic particles of the canonical problem enters the training and the testing of ANNs. After the ANNs are trained successfully, the burning rates of the reduced set of thermochemical variables are readily obtained from the knowledge of temperature and the retained species mass fractions. An Arrhenius-type reduced chemical scheme previously derived is also available for these operating conditions [35].

Direct numerical simulation (DNS) of the non-premixed syngas oxy-flame with side wall effects are then performed. First, two-dimensional DNS with the detailed chemical scheme serves as reference to test both the Arrhenius-type reduced scheme and the ANNs trained aside from DNS. Then, three-dimensional DNS of the same flame configuration are conducted with both the Arrhenius-type reduced scheme and the ANN-type chemistry. The comparison between results confirm the robustness of the ANNs for chemistry reduction along with the opportunity to train neural networks for chemistry reduction prior to flow simulations.

\section{Non-premixed flame configuration and numerical modeling}

A turbulent non-premixed oxy-flame under an elevated pressure of $341.3 \mathrm{kPa}$ with side-wall effects is considered, as shown in Fig. 1. A representative syngas composition is injected from the upper inlet over a width of $2 \mathrm{~mm}$, while pure oxygen is supplied at the lower inlet over $10 \mathrm{~mm}$. A $0.2 \mathrm{~mm}$ splitter plate separates these fuel and oxidizer inlets. The corresponding boundary 
conditions (velocity, temperature and species mass fractions) are summarized in Table 1

A preliminary simulation of a turbulent periodical 3D channel flow initialized with the oxygen bulk velocity serves as a database to impose a proper turbulent inflow for the lower oxygen stream [39, 40]. A laminar channel flow inlet profile is imposed for the close-to-wall fuel stream. A non-slip wall is placed at the top of the computational domain, while a symmetrical boundary condition is employed at the bottom. Convective boundary condition is used for the outlet of the domain. Periodic boundary conditions are used in the spanwise $z$ direction (Fig. 1). The temperature of the upper wall $T_{w}(x, t)$ is non-uniform and calculated so that the heat flux locally reaching the wall is evacuated mimicking water cooling, with a convective heat transfer $h=3300 \mathrm{~W} \cdot \mathrm{m}^{-2} \cdot \mathrm{K}^{-1}$ and a water temperature $T_{o}=353 \mathrm{~K}$ (i.e., $h\left(T_{w}-T_{o}\right)=\lambda\left(\partial T / \partial n_{w}\right)_{\text {gas }}$, where $n_{w}$ is the wall normal direction toward the gas).

The physical dimensions of the computational domain are $L_{x}=100 \mathrm{~mm}, L_{y}=12 \mathrm{~mm}$ and $L_{z}=7.68 \mathrm{~mm}$. One-dimensional canonical strained non-premixed flames simulations were conducted to calibrate the grid resolution. It was also verified through a non-reacting simulation that the structure of the wall boundary layer agrees with what is expected (viscous sub-layer, buffer layer and log-law). The mesh composed of $66.44 \mathrm{M}$ cells is uniform in the streamwise $x$ and spanwise $z$ directions, with the grid spacing $h_{x}=h_{z}=60 \mu \mathrm{m}$. In the vertical $y$ direction, the mesh keeps a fine resolution of $20 \mu \mathrm{m}$ for the upper region close to the wall $(y>10 \mathrm{~mm})$, where the flame is located. As $y$ decreases from 10 to $0 \mathrm{~mm}$, the mesh gets coarser to reach a grid spacing of $100 \mu \mathrm{m}$ at the bottom of the domain. The first point off the top wall is at $y^{+}=0.55$, where the superscript '+' denotes usual dimensionless near-wall quantities. There are 9 grid points within $y^{+}=10$ to resolve the viscous layer near the wall [41]. The time step is fixed at $0.3 \mu$ s $(\mathrm{CFL} \approx 0.8)$ with sub-iterations in a stiff-chemistry ODE solver (CVODE).

The governing equations for the gas mixture are solved with a low-Mach number in-house code [42-47]. The conservation equations for mass, momentum, species and temperature of the gas phase are cast as follows:

$$
\frac{\partial \rho}{\partial t}+\frac{\partial \rho u_{j}}{\partial x_{j}}=0,
$$




$$
\begin{gathered}
\frac{\partial \rho u_{i}}{\partial t}+\frac{\partial \rho u_{i} u_{j}}{\partial x_{j}}=-\frac{\partial p}{\partial x_{i}}+\frac{\partial \tau_{i j}}{\partial x_{j}}, \\
\frac{\partial \rho Y_{n}}{\partial t}+\frac{\partial \rho u_{j} Y_{n}}{\partial x_{j}}=\frac{\partial}{\partial x_{j}}\left(\rho D \frac{\partial Y_{n}}{\partial x_{j}}\right)+\dot{\omega}_{Y_{n}}, \\
\frac{\partial \rho T}{\partial t}+\frac{\partial \rho u_{j} T}{\partial x_{j}}=\frac{\partial}{\partial x_{j}}\left(\frac{\lambda}{C_{P}} \frac{\partial T}{\partial x_{j}}\right)+\frac{\lambda}{C_{P}^{2}} \frac{\partial C_{P}}{\partial x_{j}} \frac{\partial T}{\partial x_{j}}+\dot{\omega}_{T},
\end{gathered}
$$

where $t$ is the time [s], $\rho$ is density of the gas mixture $\left[\mathrm{kg} \cdot \mathrm{m}^{-3}\right], u$ is the gas velocity $\left[\mathrm{m} \cdot \mathrm{s}^{-1}\right]$, $Y_{n}$ is the mass fraction of the $n$-th chemical species [-], and $T$ is the gas temperature $[\mathrm{K}] . p$ is the pressure $[\mathrm{Pa}]$ and the viscous stress tensor $\tau_{i j}=\mu\left(\partial u_{i} / \partial x_{j}+\partial u_{j} / \partial x_{i}-(2 / 3)\left(\partial u_{k} / \partial x_{k}\right) \delta_{i j}\right) . \mu$, $D, \lambda$ and $C_{P}$ are the molecular viscosity $[\mathrm{Pa} \cdot \mathrm{s}]$, molecular mass diffusivity coefficient $\left[\mathrm{m}^{2} \cdot \mathrm{s}^{-1}\right]$, thermal conductivity $\left[\mathrm{W} \cdot \mathrm{m}^{-1} \cdot \mathrm{K}^{-1}\right]$ and specific heat capacity $\left[\mathrm{J} \cdot \mathrm{kg}^{-1} \cdot \mathrm{K}^{-1}\right]$ of the gas mixture, respectively. In the present study, the Lewis number is assumed to be unity for all the species, which leads to $D=\lambda /\left(\rho C_{p}\right)$; while $\mu$ and $\lambda$ are calculated using Wilke's type mixing rules. Finally, $\dot{\omega}_{Y_{n}}$ and $\dot{\omega}_{T}$ are the species and temperature source terms due to chemical reaction, respectively.

The numerical scheme is based on an approach previously employed for both DNS and largeeddy simulation (LES) [48, 49]. The time advancement relies on a second-order Crank-Nicolson scheme. However, due to the splitting method for chemistry integration, the overall order of accuracy of the solver in time advancement may drop below second-order. A bounded quadratic upstream interpolation for convective kinematics (BQUICK) scheme is applied to the scalar advection terms in the species and temperature equations, while a second-order central difference scheme is used for the scalar diffusion terms in the species and temperature equations and all terms in the momentum equation. An alternating direction implicit (ADI) approach has been used, in which semi-implicit tridiagonal/pentadiagonal equations are solved separately for each direction.

\section{Machine learning chemistry reduction methodology}

\subsection{Stochastic micro-mixing problem}

The training database for the ANN chemistry is built from a turbulent non-adiabatic nonpremixed micro-mixing canonical problem, which was first discussed for process control of selective non-catalytic reduction (SNCR) [50, 51] and then extended to automated chemistry reduc- 
tion [52].

As in the DNS of the non-premixed oxy-flame, two inlets are considered and stochastic particles evolve from initial conditions characterized by these inlets given in Table 1 . The number of stochastic particles per inlet (i.e., the mass flow rates of the inlets [50]) are organized so that the overall system operates under the stoichiometric condition. Here, a total number of 1000 stochastic particles are employed in the reactor, at initial time 660 particles are assigned to the composition and enthalpy of the fuel inlet and 340 particles to that of the oxygen inlet.

From the initial condition at time $t=0$, the species mass fraction $Y_{i}^{p}(t)$ and sensible enthalpy $h_{s}^{p}(t)$ of each stochastic particle evolve according to the following equations:

$$
\begin{aligned}
\frac{d Y_{i}^{p}(t)}{d t} & =\operatorname{MIX}_{i}^{p}\left(\tau_{T}\right)+\dot{\omega}_{i}^{p} \\
\frac{d h_{s}^{p}(t)}{d t} & =\operatorname{MIX}_{h_{s}}^{p}\left(\tau_{T}\right)+\dot{\omega}_{h_{s}}^{p}-\alpha_{\mathrm{loss}}\left(T-T_{o}\right)
\end{aligned}
$$

where 'MIX' denotes the stochastic turbulent micro-mixing closure for the diffusive budget, which is here the well-established Euclidean minimum spanning tree (EMST) mixing model [37, 53]. $\tau_{T}$ denotes the micro-mixing time and $\dot{\omega}_{i}^{p}$ and $\dot{\omega}_{h_{s}}^{p}$ are the species and enthalpy chemical sources, respectively. The linear sink term $\alpha_{\text {loss }}\left(T-T_{o}\right)$ in Eq. (6) represents convective heat loss effects, as previously done in [54, 55] in the derivation of reduced chemical schemes devoted to flame wall/interaction. $T_{o}=353 \mathrm{~K}$ is the temperature of the cooling water in the DNS configuration. $\alpha_{\text {loss }}$ is the heat loss coefficient $\left(\mathrm{W} \cdot \mathrm{kg}^{-1} \cdot \mathrm{K}^{-1}\right)$.

The value of the micro-mixing time is chosen in line with the global turbulent mixing characteristics of the shear layer. According to the inlet conditions, $\ell_{T}$, the integral length scale in the fully developed zone can be expected of the order of a few millimeters. Taking $\ell_{T} \approx 3 \mathrm{~mm}$ and velocity fluctuations $u^{\prime}$ of the order of a few meters per second, $\tau_{T} \approx \ell_{T} / u^{\prime} \sim 0.3 \mathrm{~ms}$. Accordingly, the micro-mixing time is set at $0.3 \mathrm{~ms}$ while the time step is $0.3 \mu \mathrm{s}$, with sub-iterations to solve for the stiff-chemistry part of the problem using CVODE. In fact, as soon as it does not prevent ignition or favor very fast chemistry, varying the value of $\tau_{T}$ was not found to have a major impact on the results (see [52] for a discussion on the impact of $\tau_{T}$ value on chemistry reduction using a similar stochastic formulation). In the present case, the most crucial point is to cover with Eqs. (5) 
and (6) the full range of chemical responses starting from frozen flow mixing, followed by ignition and fast oxy-flame burning, to end up with burnt products subsequently subjected to heat-loss.

Equations (5) and (6) are solved using the CANTERA package [56]. The reference GRI3.0 methane/air detailed mechanism [57] without $\mathrm{NO}_{x}$ (35 chemical species and 217 elementary reactions) serves as reference to test the reduction methodology.

\subsection{Reference reduced-scheme}

Combining a directed relation graph analysis (DRGEP) [16] with a genetic algorithm [52], an 11-species reduced scheme was previously derived with the simple pairwise Curl mixingmodel [58] and validated for the syngas oxy-flame considered here [35] (Table 22). Both the GRI-3.0 and this reduced-scheme will serve as references thereafter. The most important species found: $\mathrm{O}, \mathrm{O}_{2}, \mathrm{H}, \mathrm{H}_{2}, \mathrm{OH}, \mathrm{HO}_{2}, \mathrm{H}_{2} \mathrm{O}, \mathrm{H}_{2} \mathrm{O}_{2}, \mathrm{CO}$ and $\mathrm{CO}_{2}, \mathrm{~N}_{2}$ and the temperature are retained to constitute the reduced set of thermochemical variables.

Table 2: Reduced chemistry for syngas oxy-flame. Units are mol, $\mathrm{s}, \mathrm{cm}^{3}$, cal and $\mathrm{K}$. The Chaperon efficiencies of the GRI-3.0 mechanism are preserved for both three-body and fall-off reactions (see also supplementary material).

\begin{tabular}{|c|c|c|c|c|}
\hline $\mathrm{n}^{o}$ & Reaction & $A$ & $\beta$ & $E_{a}$ \\
\hline 1 & $2 \mathrm{O}+\mathrm{M} \rightleftharpoons \mathrm{O}_{2}+\mathrm{M}$ & $1.20 \mathrm{e}+17$ & -1.00 & 0 \\
\hline 2 & $\mathrm{O}+\mathrm{H}_{2} \rightleftharpoons \mathrm{H}+\mathrm{OH}$ & $3.87 e+04$ & 2.70 & 6260 \\
\hline 3 & $\mathrm{O}+\mathrm{HO}_{2} \rightleftharpoons \mathrm{OH}+\mathrm{O}_{2}$ & $2.00 \mathrm{e}+13$ & 0 & 0 \\
\hline 4 & $\mathrm{O}+\mathrm{CO}(+\mathrm{M}) \rightleftharpoons \mathrm{CO}_{2}(+\mathrm{M})$ & $1.80 \mathrm{e}+10$ & 0 & 2385 \\
\hline 5 & $\mathrm{O}_{2}+\mathrm{CO} \rightleftharpoons \mathrm{O}+\mathrm{CO}_{2}$ & $2.50 \mathrm{e}+12$ & 0 & 47800 \\
\hline 6 & $\mathrm{H}+\mathrm{O}_{2}+\mathrm{M} \rightleftharpoons \mathrm{HO}_{2}+\mathrm{M}$ & $2.80 \mathrm{e}+18$ & -0.86 & 0 \\
\hline 7 & $\mathrm{H}+\mathrm{O}_{2}+\mathrm{N}_{2} \rightleftharpoons \mathrm{HO}_{2}+\mathrm{N}_{2}$ & $2.60 \mathrm{e}+19$ & -1.24 & 0 \\
\hline 8 & $\mathrm{H}+2 \mathrm{O}_{2} \rightleftharpoons \mathrm{HO}_{2}+\mathrm{O}_{2}$ & $2.08 \mathrm{e}+19$ & -1.24 & 0 \\
\hline 9 & $\mathrm{H}+\mathrm{O}_{2}+\mathrm{H}_{2} \mathrm{O} \rightleftharpoons \mathrm{HO}_{2}+\mathrm{H}_{2} \mathrm{O}$ & $1.13 \mathrm{e}+19$ & -0.76 & 0 \\
\hline 10 & $\mathrm{H}+\mathrm{O}_{2} \rightleftharpoons \mathrm{O}+\mathrm{OH}$ & $2.65 e+16$ & -0.67 & 17041 \\
\hline 11 & $\mathrm{H}+\mathrm{OH}+\mathrm{M} \rightleftharpoons \mathrm{H}_{2} \mathrm{O}+\mathrm{M}$ & $2.20 \mathrm{e}+22$ & -2.00 & 0 \\
\hline 12 & $\mathrm{H}+\mathrm{HO}_{2} \rightleftharpoons \mathrm{O}_{2}+\mathrm{H}_{2}$ & $4.48 \mathrm{e}+13$ & 0 & 1068 \\
\hline 13 & $\mathrm{H}+\mathrm{HO}_{2} \rightleftharpoons 2 \mathrm{OH}$ & $8.40 \mathrm{e}+13$ & 0 & 635 \\
\hline 14 & $\mathrm{OH}+\mathrm{H}_{2} \rightleftharpoons \mathrm{H}+\mathrm{H}_{2} \mathrm{O}$ & $2.16 e+08$ & 1.51 & 3430 \\
\hline 15 & $2 \mathrm{OH}(+\mathrm{M}) \rightleftharpoons \mathrm{H}_{2} \mathrm{O}_{2}(+\mathrm{M})$ & $7.40 \mathrm{e}+13$ & -0.37 & 0 \\
\hline 16 & $\mathrm{OH}+\mathrm{HO}_{2} \rightleftharpoons \mathrm{O}_{2}+\mathrm{H}_{2} \mathrm{O}$ & $1.45 \mathrm{e}+13$ & 0 & -500 \\
\hline 17 & $\mathrm{OH}+\mathrm{H}_{2} \mathrm{O}_{2} \rightleftharpoons \mathrm{HO}_{2}+\mathrm{H}_{2} \mathrm{O}$ & $2.00 \mathrm{e}+12$ & 0 & 427 \\
\hline 18 & $\mathrm{OH}+\mathrm{H}_{2} \mathrm{O}_{2} \rightleftharpoons \mathrm{HO}_{2}+\mathrm{H}_{2} \mathrm{O}$ & $1.70 \mathrm{e}+18$ & 0 & 29410 \\
\hline 19 & $\mathrm{OH}+\mathrm{CO} \rightleftharpoons \mathrm{H}+\mathrm{CO}_{2}$ & $4.76 e+07$ & 1.23 & 70 \\
\hline 20 & $2 \mathrm{HO}_{2} \rightleftharpoons \mathrm{O}_{2}+\mathrm{H}_{2} \mathrm{O}_{2}$ & $1.30 \mathrm{e}+11$ & 0 & -1630 \\
\hline 21 & $2 \mathrm{HO}_{2} \rightleftharpoons \mathrm{O}_{2}+\mathrm{H}_{2} \mathrm{O}_{2}$ & $4.20 \mathrm{e}+14$ & 0 & 12000 \\
\hline 22 & $\mathrm{HO}_{2}+\mathrm{CO} \rightleftharpoons \mathrm{OH}+\mathrm{CO}_{2}$ & $1.50 \mathrm{e}+14$ & 0 & 23600 \\
\hline 23 & $\mathrm{OH}+\mathrm{HO}_{2} \rightleftharpoons \mathrm{O}_{2}+\mathrm{H}_{2} \mathrm{O}$ & $5.00 \mathrm{e}+15$ & 0 & 17330 \\
\hline
\end{tabular}

\subsection{Non-premixed database for ANN training}

At the start of the simulation with the detailed scheme (GRI-3.0), $\alpha_{\text {loss }}$ is set to zero in Eq. (6), i.e., no heat loss is present. Because the particles of the fuel inlet are preheated to $1223 \mathrm{~K}$, rapid 
ignition occurs with the detailed chemistry mechanism at $81 \mu$ s after mixing with the particles from the oxygen inlet. Figure 2 shows representative scatter plots of the instantaneous temperature of the stochastic particles against mixture fraction. The instantaneous values at a given time (red) are plotted along with the time history of all particles up to this time, for three representative times. The mixture fraction is defined as $Z=Y_{\mathrm{N}_{2}} / 0.1549$. Because of fuel preheating (Table 1), ignition first appears on the fuel-rich side for $Z>0.9$ (Fig. 2(a)). After $0.3 \mathrm{~ms}$, i.e., 1000 time steps, the micro-mixing simulation reaches a stable burning status. The peak temperature is $3113 \mathrm{~K}$ around the stoichiometric condition at $Z=0.66$ (Fig. 2(b) . Then, to model the interaction of burnt gases with a cold wall, the heat loss is turned on by setting $\alpha_{\text {loss }}=10^{7} \mathrm{~W} \cdot \mathrm{kg}^{-1} \cdot \mathrm{K}^{-1}$, and the simulation proceed for another $0.6 \mathrm{~ms}$ until all the stochastic particles are cooled down (Fig. 2(c)).

By saving the 35 species mass fractions and temperature of each stochastic particle for every time step, 3000 snapshots each containing thermochemical information from 1000 particles are available to built a training database. To augment the database up to 9000 snapshots and avoid over-fitting, as it is usually done in ANN training procedure, the 3,000,000 data are perturbed twice applying $2 \%$ and $3 \%$ of uncorrelated random noise, still securing mass conservation.

The chemical sources of intermediate radicals featuring the fastest evolution (i.e., those associated to the smallest time scales) are known to be difficult to interpolate, because the concentrations of these species jump from zero to their maximum levels over very small characteristic times. The issue appeared in the present case for the evolution of $\mathrm{H}_{2} \mathrm{O}_{2}$, a species which was found mandatory to be kept in the reduced scheme to properly capture ignition. To secure an accurate interpolation of the $\mathrm{H}_{2} \mathrm{O}_{2}$ source with the ANN, the neural network needs to be exposed to numerous data points, where the $\mathrm{H}_{2} \mathrm{O}_{2}$ concentration lies between zero and its maximum. A brute force way to overcome this difficulty would be to train the ANN at all the sub-iterations of the time integration performed by the stiff-chemistry solver (CVODE), which returns the proper evolutions of the radical species entering the detailed scheme. This option would result in a huge computing effort during the learning phase.

Instead, an alternative data driven approach has been followed, which is valid only for a single variable affected by an overly quick evolution and whose contribution to the overall mass stays small (the max value of $\mathrm{H}_{2} \mathrm{O}_{2}$ mass fraction is $5.3 \times 10^{-4}$ ). To populate the training database with 
a sufficient variety of $\mathrm{H}_{2} \mathrm{O}_{2}$ chemical sources, fields are manufactured by adding a copy of the 9000 snapshots in which the $\mathrm{H}_{2} \mathrm{O}_{2}$ mass fraction is replaced by a random value between zero and its peak level. The mass fractions of others species are scaled according to the new total mass fractions, to conserve mass. These species vectors with specific $\mathrm{H}_{2} \mathrm{O}_{2}$ mass fractions then enter the GRI-3.0 mechanism, to compute their corresponding exact chemical sources. For these additional species vectors, the detailed scheme returns chemical source rates which fulfill all the expected physical properties imposed by the detailed scheme and which can be used for the neural network to learn the relation between species mass fraction and temperature, as input, and sources, as output. Hence, the training of the ANN to reproduce chemical sources with intermediate values of $\mathrm{H}_{2} \mathrm{O}_{2}$ becomes easily tractable.

The database now have 18000 snapshots each containing 1000 data. $2.7 \%$ of the data have a temperature below $320 \mathrm{~K}$, these points with negligible sources are dropped out, which leads to a final database of $17.5 \times 10^{6}$ data.

\subsection{ANN training procedure}

Figure 3 shows the overall ANN training procedure from the micro-mixing database. The input of the ANNs are the vectors $\underline{\phi}(t)$ of the 10 species mass fractions and temperature $\left(\mathrm{N}_{2}\right.$ not included). The "target" (or "label") of each input vector is the corresponding reaction rates $\dot{\omega}_{i}=$ $\left(\phi_{i}\left(t+\delta t_{o}\right)-\phi_{i}(t)\right) / \delta t_{o}$, with $\delta t_{o}=0.3 \mu \mathrm{s}$, the time step of flow simulations in the present work (close to the shortest chemical time scale observed for the intermediate species retained in the mechanism). Organizing the sources in this form is convenient for the subsequent coupling with flow simulation following two options: In the case where a specific time integration method for stiff differential systems is preferred, the source can be used directly. If sub-iterations take place with the time step $\delta t>\delta t_{o}$, interpolations will be needed to estimate $\dot{\omega}_{i}(t)$. A second option for the coupling with the flow, is to directly read from the ANNs $\phi_{i}(t+\delta t)$. Here again, if $\delta t>\delta t_{o}$, sub-iterations will be required to secure accuracy. The second option is used thereafter and since $\delta t=\delta t_{o}$ in our flow simulation, sub-iterations are not performed.

The chemical sources of the reduced set of $N_{S}^{r}=11$ species (source of $\mathrm{N}_{2}$ is set to zero) are computed from the detailed chemical scheme with the $N_{S}=35$ species. Therefore these 
sources do not preserve the atomic budgets computed over the $N_{S}^{r}<N_{S}$ species. Even if at its maximum, the mass default is only $0.016 \%$ of the total mass of these atoms, in order to fully conserve mass, corrective sources are added according to the relative contribution of the species to atomic budgets. Let us denote $\alpha_{A, i}$ the number of atom $\mathrm{A}$ (i.e., $\mathrm{A} \equiv \mathrm{C}, \mathrm{H}$ or $\mathrm{O}$ ) in the $i$-th species. The mass imbalance in A source reads for the reduced set of species

$$
\Delta \dot{\omega}_{\mathrm{A}}=\sum_{i=1}^{N_{s}^{r}} \frac{\alpha_{\mathrm{A}, i} W_{\mathrm{A}}}{W_{i}} \dot{\omega}_{i},
$$

where $W_{\mathrm{A}}$ and $W_{i}$ are molar weights. Sources of carbon containing species of the syngas (CO and $\left.\mathrm{CO}_{2}\right)$ and hydrogen containing species $\left(\mathrm{H}_{2}, \mathrm{H}, \mathrm{H}_{2} \mathrm{O}, \mathrm{OH}, \mathrm{HO}_{2}\right.$ and $\left.\mathrm{H}_{2} \mathrm{O}_{2}\right)$ are corrected adding

$$
\Delta \dot{w}_{i}=-\frac{Y_{i}}{Y_{\mathrm{A}}} \Delta \dot{\omega}_{\mathrm{A}}
$$

with the atom mass fraction $Y_{\mathrm{A}}=\sum_{i=1}^{N_{s}^{r}} \alpha_{\mathrm{A}, i}\left(W_{A} / W_{i}\right) Y_{i}$. For oxygen, Eq. 8 is applied only to $\mathrm{O}_{2}$ and with the already corrected sources of $\mathrm{C}$ and $\mathrm{H}$ containing species. This correction is applied during the ANN-DNS coupled simulations (therefore after ANN training). Then, the chemical sources perfectly preserve atomic budgets, as chemical sources computed from elementary reactions with Arrhenius laws would. In practice this correction stays marginal, a source correction (Eq. (8)) significantly contributing to the burning rates would mean that too many species were removed, jeopardizing the quality of the ANN prediction.

Within the information from the stochastic particles containing $17.5 \times 10^{6}$ data, $3.5 \times 10^{6}(20 \%)$ of them are randomly selected to form the testing database, while the remaining $14 \times 10^{6}(80 \%)$ constitute the training database. All data contributing to the "input vectors" and to the "targets" are normalized by their respective maximum absolute value over the entire database, and these maximum levels are stored. 
Table 3: Structure of the sequential ANNs used.

\begin{tabular}{lll}
\hline Layer type & $\begin{array}{l}\text { Activation } \\
\text { function }\end{array}$ & Output Shape \\
\hline Input & - & (None, 11) \\
Dense & ReLU & (None, 512) \\
Dense & ReLU & (None, 256) \\
Dense & ReLU & (None, 128) \\
Dense & ReLU & (None, 64) \\
Dense & ReLU & (None, 16) \\
Dense (Output) & - & (None, 11) \\
\hline
\end{tabular}

\subsection{ANN structure}

The exact structure of the sequential ANNs 1 is summarized in Table 3 . Five fully connected dense layers are employed between the input and output layers. Each neuron of the dense layers 2 , (see green circles in Fig. 3) performs the following two operations: a weighted sum of inputs from all the neurons of the previous layer and the subsequent calculation of a non-linear activation function. In the present study, the popular rectified linear unit (ReLU) activation function is used:

$$
\operatorname{ReLU}(x)=\max (x, 0)
$$

Various options were attempted (even using a different network for each species and temperature). In the end, the above ANN topology, which is a quite standard one, including the number of layers and neurons per layer, appears as the simplest efficient network for the prediction of the chemical sources. More complex networks would slow down the training process, with an increased risk of overfitting. In total, the neural network contains 179,851 weights to be adjusted.

To improve the precision of the ANNs for small values of the chemical sources, a second network with the exact same structure is trained for a subset of the database defined by $Y_{\mathrm{O}_{2}}>0.9$, i.e., where the overall burning rates stay very low. A sufficiently large number of data points are involved in the training so that continuity is secured between the two networks. The subset-

\footnotetext{
${ }^{1}$ In a sequential ANN, each layer has exactly one input tensor and one output tensor.

${ }^{2} \mathrm{~A}$ dense layer is driven by the same formulas as the linear layers, but the end result is passed through a non-linear function called 'Activation function'.
} 
database is normalized by its own maximum absolute values. In the following, the networks are referred to as the main ANN and the second ANN (subset for lower burning rates).

The training of the ANNs is performed using the TensorFlow Python library with GPU support (www.tensorflow.org). The neural weights are adjusted during the training process until a satisfying minimal error is obtained between the "target" sources and the ones predicted by the ANNs. The Adam optimizer [59] is used with a mean-squared-error (MSE) loss function comparing the predictions to the target. A training step is performed with a batch of 10240 "input vectors", leading to 1369 steps per "epoch"/iteration for the main ANN. The learning rate [60] is initially set to 0.0003 , to decay versus the steps $(n)$ according to

$$
l_{r}=\frac{0.0003}{1.0+0.0001 \cdot n} .
$$

The network is trained for 500 epochs to reach convergence, which requires for the main ANN about 3.2 hours on an Nvidia GeForce GTX 1080 Ti GPU.

\subsection{ANN testing}

The performance of the ANNs is first evaluated a-priori on the testing database containing $3.5 \times 10^{6}$ data unseen during training. The mean-square error between the ANN predictions and the targets is $9.2 \times 10^{-7}$ for the main ANN and $3.6 \times 10^{-7}$ for the second ANN. Figure 4 shows the predicted chemical sources against their target values for both the main ANN and the second ANN, confirming in a preliminary manner the accuracy of the ANN reduced chemistry approach.

\section{ANN-DNS full coupling}

The ANN reduced chemistry is now coupled with the flow solver to simulate the syngas nonpremixed oxy-flame (only CPU is used in this part with ANNs exported in C language, see supplementary material). To evaluate the performance of the ANN reduced chemistry, simulations are performed with the reference detailed GRI-3.0 chemistry (2D), ANN chemistry (2D and 3D) and the reduced chemistry of Table 2 (2D and 3D). 


\subsection{Detailed, Arrhenius-reduced and ANN-reduced chemistry in 2D simulations}

The comparison of the 2D DNS results between the detailed GRI-3.0, the 11-species reduced mechanism of Table 2 and the ANN chemistry are shown in Figs. 5 and 6 (streamwise and spanwise distributions). Statistical averages have been collected for 10 flow-through times after the flow is fully established.

Until the position $x \approx 8 \mathrm{~mm}$ along the planar fuel jet centerline (Fig. 5), the averaged streamwise velocity distribution decreases. At this streamwise location, the non-premixed syngas/air mixture starts to ignite, as indicated by the increase in temperature, while $\mathrm{CO}$ and $\mathrm{H}_{2}$ decrease to produce $\mathrm{CO}_{2}$ and $\mathrm{H}_{2} \mathrm{O}$ (Fig. 5)(a)). At $15 \mathrm{~mm}$ from the nozzle inlet, approaching the cooled wall, the chemistry slows down ( $x=0$ in Fig. 6(a)). From this streamwise position the flame progressively spreads, to approach the close vicinity of the wall and the plate progressively heats up (Fig. 6(c)). Overall, the system is fuel-lean and the mass fraction of $\mathrm{O}_{2}$ increases along the centerline. $\mathrm{O}$ and $\mathrm{OH}$ are the two major radicals while the mass fractions of $\mathrm{HO}_{2}$ and $\mathrm{H}_{2} \mathrm{O}_{2}$ are well below $10^{-4}$ (Fig. 5(b)).

Comparing against the simulation with the reference detailed GRI-3.0 chemistry mechanism (circles in Figs. 5 and 6), velocity, temperature and all the major species are well captured by the ANN-DNS (dashed-lines). Considering the radicals and minor species, the ANN-DNS shows a good prediction on the two major radicals $\mathrm{O}$ and $\mathrm{OH}$, while it tends to over-predict $\mathrm{HO}_{2}$ and under-predict $\mathrm{H}_{2} \mathrm{O}_{2}$.

The statistics from the simulation with the reduced mechanism of Table 2(lines) are also very close to the detailed chemistry DNS and will serve as reference to test the ANN in 3D-DNS. For the $\mathrm{H}$ radical, the ANN-DNS returns a closer agreement against the reference detailed mechanism (Figs. 5(b) and 6). Because of the large number of sub-steps involved in the generation of both the ANN chemistry and the reduced mechanism of Table 2, it is difficult to identify the exact underlying reason for this behavior of the $\mathrm{H}$ radical. This is one of the weak point of data driven approaches, in the forest of neural connections and weights, it is barely feasible to track back specific relations between the species which lead to the behavior of intermediate minor species. However, it is likely that learning from the large number of information available during the training phase, was helpful for the prediction of radicals. Notice also that the ANNs were trained with 
the detailed mechanism and the errors are due to the machine learning procedure (training dataset, training method, etc.). On the other hand, the reduced mechanism has been derived by dropping some species and reactions and therefore its discrepancies are for totally different reasons.

\subsection{Arrhenius-reduced and ANN chemistry in 3D simulations}

Vortex stretching was missing in the two-dimensional simulations, therefore the statistics collected in the three-dimensional flow are different from those of the planar flow. In particular, slightly smoother gradients are observed in $3 \mathrm{D}$, as seen in Figs. 7 and 8 , compared to Figs. 5 and 6. Overall, the three-dimensional turbulent flow cascade enhances the turbulence-chemistry interaction, leading to different scalar averages.

The ANN-chemistry provides statistics which are very close to those obtained with the reduced mechanism, for all the major species (Figs. 7 and 8 ). A departure is observed for $\mathrm{H}$ and also for $\mathrm{HO}_{2}$ and $\mathrm{H}_{2} \mathrm{O}_{2}$. The ANN chemistry predicts a higher level for $\mathrm{HO}_{2}$ and a lower level for $\mathrm{H}$ and $\mathrm{H}_{2} \mathrm{O}_{2}$. According to the comparison against the GRI-3.0 reported above in the two-dimensional case, the scalar structure of $\mathrm{H}$ radical obtained with the ANN chemistry is actually expected to be closer than the reduced scheme to the one that would be seen in 3D with the detailed mechanism, which would be too expensive with available computing power. At stage, it is concluded that for the limited amount of species involved, the ignition phenomenon and the responses of reactants and major products are well captured, which is the main objective of most reduced chemical schemes used in computational fluid dynamics of complex systems. However as it can be expected, much more degrees of freedom would be needed to fully predict radicals and intermediate species.

Figure 9 shows the statistics of wall heat flux at the upper wall for both $2 \mathrm{D}$ and 3D cases, good agreement is found between ANN chemistry and reduced chemistry. Right after the splitter plate, the wall heat flux shows a high value of about $800 \mathrm{~kW} \cdot \mathrm{m}^{-2}$ at $x=0$, which is due to the injection of the preheated fuel at $1223 \mathrm{~K}$. As the fuel gets cooled down by the water-cooled wall, the mean heat flux decreases rapidly to the order of $200 \mathrm{~kW} \cdot \mathrm{m}^{-2}$ at $x=30 \mathrm{~mm}$ in the $2 \mathrm{D}$ cases (Fig. 9(a)). Then, the mean heat flux starts to increase until $x=80 \mathrm{~mm}$, where it reaches its maximum of $1250 \mathrm{~kW} \cdot \mathrm{m}^{-2}$, because of the flame-wall interaction. Further downstream, the mean heat flux decreases again when the flame losses heat at the wall. The intermittency of the 
flame approaching and leaving the wall promotes large fluctuations levels for $x \in[50 \mathrm{~mm}, 90 \mathrm{~mm}]$ (Fig. 9(a)). The heat flux profiles feature similar characteristics in 3D, with however turbulent mixing in the third direction leading to a delayed increase of the mean heat flux and also a lower peak value at about $900 \mathrm{~kW} \cdot \mathrm{m}^{-2}$. This behavior is confirmed in the mean temperature distribution shown in Fig. 10, as expected the thermal boundary layer in 2D case is much thinner than that in 3D case.

The computational cost of the coupled ANN-DNS approach is given in Fig. 11 for the 2D simulations, which include all three approaches (detailed, reduced and ANN). For solving the chemical source part of the problem, simulations with ANN chemistry are 25 times faster than those with the GRI-3.0 detailed mechanism, and 3 times faster than the 11-species reduced mechanism. This speed-up results mainly from the fact that Arrhenius rates calculation are not needed and the time integration of stiff chemical sources is also avoided when the option of directly reading the species increments from the ANN is chosen. The computational time for solving the convectivediffusive part of the turbulent flame mainly depends on the number of species to be transported, it is therefore similar for the ANN-DNS and reduced chemistry DNS. (Same remarks apply to the 3D-DNS.)

\section{Conclusions}

One of the major stumbling block to the systematic introduction of artificial neural networks (ANNs) in flame simulation lies in the definition of generic training procedures, so that actually performing the simulation of the target flow geometry is not mandatory to complete the training of the ANN. Canonical problems, associated to given network architectures and training procedures, which could be applied prior to flame simulations, are likely to help popularizing the application of machine learning to computational combustion. Indeed, aside from a few pioneer works [26, 36], in most of previously published applications, the training and the subsequent use of the neural networks have been demonstrated on the very same flame configuration, thus limiting the generic character of the approach.

In the present work, a non-adiabatic non-premixed canonical problem, based on a stochastic micro-mixing closure, was introduced to train artificial neural networks from a reference detailed 
chemistry (GRI-3.0), including heat-loss effects. The trained ANNs provide directly the reaction rates of a reduced set of chemical species from the knowledge of their mass fractions and temperature, thus reducing drastically the cost of chemistry simulation. The training, performed aside from the flow simulation, is based on a reference detailed mechanism involving a large number of intermediates species and elementary reactions. This secures that the ANNs benefit from the fully detailed thermochemical information to predict the burning rate of a limited number of chemical species, which will be transported with the flow in the three-dimensional unsteady simulation.

The ANN reduced chemistry is applied to the direct numerical simulation of a non-premixed turbulent syngas-flame interacting with a cooled-wall. To assess the quality of the ANN reduced chemistry, two-dimensional simulations are simultaneously conducted with the reference fully detailed scheme (GRI-3.0) and an 11-species chemical mechanism specifically reduced for the syngas operating conditions considered. The three simulations return very similar results, with the ANN chemistry providing a significant reduction in CPU cost (25 times faster than detailed chemistry and 3 time faster than the reduced scheme). Then, the ANN chemistry and the reduced chemistry are applied to a three-dimensional direct numerical simulation of the syngas-flame with side wall effects. The statistical analysis of the non-premixed turbulent flame confirms the good performance of the ANN reduced chemistry trained prior and aside from the flow simulation. The scalar flame structure, in terms of reactants and major products, and the wall heat-flux are well predicted by the ANN reduced chemistry. Most of the expected trends are also recovered for the intermediate radicals. The potential of the discussed data driven approach lies in the automated character of the procedure to derive the reduced scheme, which can easily be implemented as a pre-processing tool. Another major advantage is that both the Arrhenius rates calculation and the direct integration of the stiff chemical system are avoided when choosing the option of reading the species increments directly from the ANN, which therefore leads to a significant reduction in CPU cost.

More physics could be introduced in the non-premixed canonical problem, as liquid fuel injection [50, 61] or multiple inlets [52], however the extension to turbulent premixed combustion would require additional work to account for flame propagation. 


\section{Acknowledgments}

This work is supported by ADEME (Agence de l'Environnement et de la Maîtrise de l'Energie) under the project IGAR (Injection de Gaz de Recycle) Grant Nb 1882C001. Computing resources were provided by CRIANN (http://www.criann.fr).

\section{References}

[1] N. Peters, Systematic reduction of flame kinetics: Principles and Details, Progress in Astronautics and Aeronautics (113) (1988) 67-86.

[2] N. Peters, Flame Calculations with Reduced Mechanisms - An Outline, in: N. Peters, B. Rogg (Eds.), Reduced Kinetic Mechanisms for Applications in Combustion Systems, Vol. 15 of Lecture Notes in Physics, SpringerVerlag Berlin Heidelberg, 1993, pp. 3-14.

[3] J. F. Griffiths, Reduced kinetic models and their application to practical combustion systems, Prog. Energy Combust. Sci. 21 (1).

[4] D. A. Goussis, U. Maas, Model Reduction for Combustion Chemistry, in: T. Echekki, E. Mastorakos (Eds.), Turbulent Combustion Modeling: Advances, New Trends and Perspectives, Springer Netherlands, Dordrecht, 2011, pp. 193-220.

[5] T. Turanyi, A. S. Tomlin, Analysis of Kinetic Reaction Mechanisms, Springer, Berlin, 2014.

[6] H. J. Curran, Developing detailed chemical kinetic mechanisms for fuel combustion, Proc. Combust. Inst. 37 (1) (2019) 57-81.

[7] N. Peters, Numerical and asymptotics analysis of systematically reduced reaction schemes for hydrocarbon flames, in: Numerical simulation of combustion phenomena, Springer-Verlag, Berlin, 1985, pp. 90-109.

[8] W. P. Jones, R. P. Lindstedt, Global reaction schemes for hydrocarbon combustion, Combust. Flame 73 (1988) 233-249.

[9] D. Bradley, L. K. Kwa, A. K. C. Lau, M. Missaghi, S. B. Chin, Laminar flamelet modeling of recirculating premixed methane and propane-air combustion, Combust. Flame 71 (2) (1988) 109-122.

[10] U. Maas, S. Pope, Simplifying chemical kinetics: Intrinsic low-dimensional manifolds in composition space, Combust. Flame 88 (3) (1992) 239-264.

[11] S. H. Lam, D. A. Goussis, The CSP method for simplifying kinetics, Int. J. Chem. Kinet. 26 (4) (1994) $461-486$.

[12] J. A. van Oijen, F. A. Lammers, L. P. H. de Goey, Modeling of complex premixed burner systems by using flamelet-generated manifolds, Combustion and Flame 127 (3) (2001) 2124-2134.

[13] V. Bykov, U. Maas, The extension of the ILDM concept to reaction-diffusion manifolds, Combust. Theory Model. 11 (6) (2007) 839-862. 
[14] W. P. Jones, S. Rigopoulos, S. Smith, U. Maas, Reduction of comprehensive chemistry via constraint potentials, Proc. Combust. Inst. 30 (1) (2003) 1325-1331.

[15] T. Lu, C. K. Law, A directed relation graph method for mechanism reduction, Proceedings of the Combustion Institute 30 (1) (2005) 1333 - 1341.

[16] P. Pepiot-Desjardins, H. Pitsch, An efficient error-propagation-based reduction method for large chemical kinetic mechanisms, Combustion and Flame 154 (1) (2008) 67 - 81.

[17] J. Sutherland, A. Parente, Combustion modeling using principal component analysis, Proc. Combust. Inst. 32 (1) (2009) 1563-1570.

[18] P. Boivin, C. Jiménez, A. L. Sánchez, F. A. Williams, An explicit reduced mechanism for $\mathrm{H}_{2}$ air combustion, Proc. Combust. Inst. 33 (1) (2011) 517 - 523.

[19] N. Jaouen, L. Vervisch, P. Domingo, Auto-thermal reforming (ATR) of natural gas: An automated derivation of optimised reduced chemical schemes, Proceedings of the Combustion Institute 36 (3) (2017) 3321 - 3330.

[20] P. Koniavitis, S. Rigopoulos, W. Jones, Reduction of a detailed chemical mechanism for a kerosene surrogate via RCCE-CSP, Combustion and Flame 194 (2018) 85 - 106.

[21] Y. Chang, M. Jia, B. Niu, Z. Xu, Z. Liu, Y. Li, M. Xie, Construction of a skeletal oxidation mechanism of n-pentanol by integrating decoupling methodology, genetic algorithm, and uncertainty quantification, Combust. Flame 194 (2018) 15-27.

[22] A. Scholtissek, P. Domingo, L. Vervisch, C. Hasse, A self-contained composition space solution method for strained and curved premixed flamelets, Combust. Flame. 207 (2019) 342-355.

[23] X. Wen, Y. Luo, H. Wang, K. Luo, H. Jin, J. Fan, A three mixture fraction flamelet model for multi-stream laminar pulverized coal combustion, Proc. Combust. Inst. 37 (3) (2019) 2901-2910.

[24] F. Christo, A. Masri, E. Nebot, S. Pope, An integrated PDF/neural network approach for simulating turbulent reacting systems, Symp. (Int.) Combust. 26 (1) (1996) 43-48.

[25] J. A. Blasco, N. Fueyo, C. Dopazo, J. Ballester, Modelling the temporal evolution of a reduced combustion chemical system with artificial neural network, Combust. Flame 113 (1-2) (1998) 38-52.

[26] L. L. Franke, A. K. Chatzopoulos, S. Rigopoulos, Tabulation of combustion chemistry via Artificial Neural Networks (ANNs): Methodology and application to LES-PDF simulation of Sydney flame L, Combust. Flame 185 (2017) 245-260.

[27] F. R. R. Padilha, A. L. D. Bortoli, Solutions for a laminar jet diffusion flame of methly formate using a skeletal mechanism obtained by applying ANNs, Journal of Mathematical Chemistry 57 (2019) 2229-2247.

[28] O. Owoyele, P. Kundu, M. M. Ameen, T. Echekki, S. Som, Application of deep artificial neural networks to multi-dimensional flamelet libraries and spray flames, International Journal of Engine Research 21 (1) (2020) 151-168.

[29] R. Ranade, T. Echekki, A framework for data-based turbulent combustion closure: A posteriori validation, 
Combustion and flame 210 (2019) 279-291.

[30] G. Aversano, A. Bellemans, Z. Li, A. Coussement, O. Gicquel, A. Parente, Application of reduced-order models based on PCA \& Kriging for the development of digital twins of reacting flow applications, Computers \& Chemical Engineering 121 (2) (2019) 422-441.

[31] Z. Nikolaou, C. Chrysostomou, L. Vervisch, R. S. Cant, Progress Variable Variance and Filtered Rate Modelling Using Convolutional Neural Networks and Flamelet Methods, Flow Turbulence Combust. 103 (2) (2019) 485501.

[32] C. J. Lapeyre, A. Misdariis, N. Cazard, D. Veynante, T. Poinsot, Training convolutional neural networks to estimate turbulent sub-grid scale reaction rates, Combust. Flame 203 (2019) 255-264.

[33] A. Seltz, P. Domingo, L. Vervisch, Z. M. Nikolaou, Direct mapping from LES resolved scales to filtered-flame generated manifolds using convolutional neural networks, Combust. Flame 210 (2019) 71-82.

[34] X. Zhu, Z. Cai, J. Wu, Y. Cheng, Q. Huang, Convolutional neural network based combustion mode classification for condition monitoring in the supersonic combustor, Acta Astronautica 159 (2019) 349-357.

[35] K. D. Wan, C. Barnaud, L. Vervisch, P. Domingo, Machine learning for detailed chemistry reduction in DNS of a syngas turbulent oxy-flame with side-wall effects, Proc. Combust. Inst. 38, in press.

[36] A. K. Chatzopoulos, S. Rigopoulos, A chemistry tabulation approach via Rate Controlled Constrained Equilibrium (RCCE) and Artificial Neural Networks (ANNs), with application to turbulent non-premixed $\mathrm{CH}_{4} / \mathrm{H}_{2} / \mathrm{N}_{2}$ flames, Proceedings of the Combustion Institute 34 (1) (2013) 1465-1473.

[37] S. Subramaniam, S. B. Pope, A mixing model for turbulent reactive flows based on Euclidean minimum spanning trees, Combustion and Flame 115 (4) (1998) 487 - 514.

[38] J. C. R. Hunt, A. A. Wray, P. Moin, Eddies, streams, and convergence zones in turbulent flows, Center for Turbulence Research Proceedings of the Summer Program CTR-S88 (1988) 193-208.

[39] C. D. Pierce, P. Moin, Method for generating equilibrium swirling inflow conditions, AIAA journal 36 (7) (1998) $1325-1327$.

[40] J. U. Schlüter, H. Pitsch, P. Moin, Large-eddy simulation inflow conditions for coupling with Reynolds-averaged flow solvers, AIAA journal 42 (3) (2004) 478-484.

[41] A. Gruber, R. Sankaran, E. R. Hawkes, J. H. Chen, Turbulent flamewall interaction: a direct numerical simulation study, J. Fluid Mech. 658 (2010) 5-32.

[42] K. D. Wan, L. Vervisch, J. Xia, P. Domingo, Z. H. Wang, Y. Z. Liu, K. F. Cen, Alkali metal emissions in an early-stage pulverized-coal flame: DNS analysis of reacting layers and chemistry tabulation, Proceedings of the Combustion Institute 37 (3) (2019) 2791-2799.

[43] K. D. Wan, Z. H. Wang, J. Xia, L. Vervisch, P. Domingo, Y. Lv, Y. Z. Liu, Y. He, K. F. Cen, Numerical study of $\mathrm{HCl}$ and $\mathrm{SO} 2$ impact on sodium emissions in pulverized-coal flames, Fuel 250 (2019) $315-326$.

[44] K. D. Wan, Z. H. Wang, J. Xia, L. Vervisch, P. Domingo, Y. Lv, Y. Z. Liu, Y. He, K. F. Cen, Numerical study of 
$\mathrm{HCl}$ and $\mathrm{SO} 2$ impact on potassium emissions in pulverized-biomass combustion, Fuel Processing Technology 193 (2019) $19-30$.

[45] K. D. Wan, J. Xia, L. Vervisch, Y. Z. Liu, Z. H. Wang, K. F. Cen, Modelling alkali metal emissions in large-eddy simulation of a preheated pulverised-coal turbulent jet flame using tabulated chemistry, Combustion Theory and Modelling 22 (2) (2018) 203-236.

[46] K. D. Wan, J. Xia, Z. H. Wang, M. Pourkashanian, K. F. Cen, Large-eddy Simulation of Pilot-assisted Pulverized-coal Combustion in a Weakly Turbulent Jet, Flow, Turbulence and Combustion 99 (2) (2017) 531550.

[47] K. D. Wan, J. Xia, Z. H. Wang, L. C. Wrobel, K. F. Cen, Online-CPD-coupled large-eddy simulation of pulverized-coal pyrolysis in a hot turbulent nitrogen jet, Combustion Science and Technology 189 (1) (2017) 103-131.

[48] C. D. Pierce, P. Moin, Progress-variable approach for large-eddy simulation of non-premixed turbulent combustion, Journal of Fluid Mechanics 504 (2004) 73-97.

[49] O. Desjardins, G. Blanquart, G. Balarac, H. Pitsch, High order conservative finite difference scheme for variable density low Mach number turbulent flows, Journal of Computational Physics 227 (15) (2008) 7125-7159.

[50] B. Farcy, L. Vervisch, P. Domingo, N. Perret, Reduced-order modeling for the control of selective non-catalytic reduction (SNCR), AIChE Journal 62 (3) (2016) 928-938.

[51] C. Locci, L. Vervisch, B. Farcy, N. Perret, Selective Non-Catalytic Reduction (SNCR) of nitrogen oxide emissions: A perspective from numerical modeling, Flow Turbulence and Combust. 100 (2) (2018) 301-340.

[52] N. Jaouen, L. Vervisch, P. Domingo, G. Ribert, Automatic reduction and optimisation of chemistry for turbulent combustion modelling: Impact of the canonical problem, Combustion and Flame 175 (2017) 60-79.

[53] Z. Ren, S. Subramaniam, S. B. Pope, Implementation of the EMST mixing model, http://tcg.mae.cornell. edu/emst (2002).

[54] K. Bioche, L. Vervisch, G. Ribert, Premixed flamewall interaction in a narrow channel: impact of wall thermal conductivity and heat losses, Journal of Fluid Mechanics 856 (2018) 535.

[55] K. Bioche, G. Ribert, L. Vervisch, Simulating upstream flame propagation in a narrow channel after wall preheating: Flame analysis and chemistry reduction strategy, Combustion and Flame 200 (2019) 219 - 231.

[56] D. G. Goodwin, R. L. Speth, H. K. Moffat, B. W. Weber, Cantera: An Object-oriented Software Toolkit for Chemical Kinetics, Thermodynamics, and Transport Processes, https://www. cantera.org, version 2.4.0 (2018).

[57] G. P. Smith, D. M. Golden, M. Frenklach, N. W. Moriarty, B. Eiteneer, M. Goldenberg, C. T. Bowman, R. K. Hanson, S. Song, W. C. Gardiner Jr., V. V. Lissianski, Z. Qin, GRI-Mech 3.0, http://www.me.berkeley. edu/gri_mech/(1999).

[58] R. L. Curl, Dispersed phase mixing: I. Theory and effects in simple reactors, AIChE Journal 9 (2) (1963) 175- 
181.

[59] D. P. Kingma, J. Ba, Adam: A Method for Stochastic Optimization, arXiv e-prints (2014) arXiv:1412.6980.

[60] H. H. Aghdam, E. J. Heravi, Guide to convolutional neural networks, Springer, New York, 2017.

[61] A. Bouaniche, N. Jaouen, P. Domingo, L. Vervisch, Vitiated High Karlovitz n-decane/air Turbulent Flames: Scaling Laws and Micro-mixing Modeling Analysis, Flow Turbulence and Combust. 


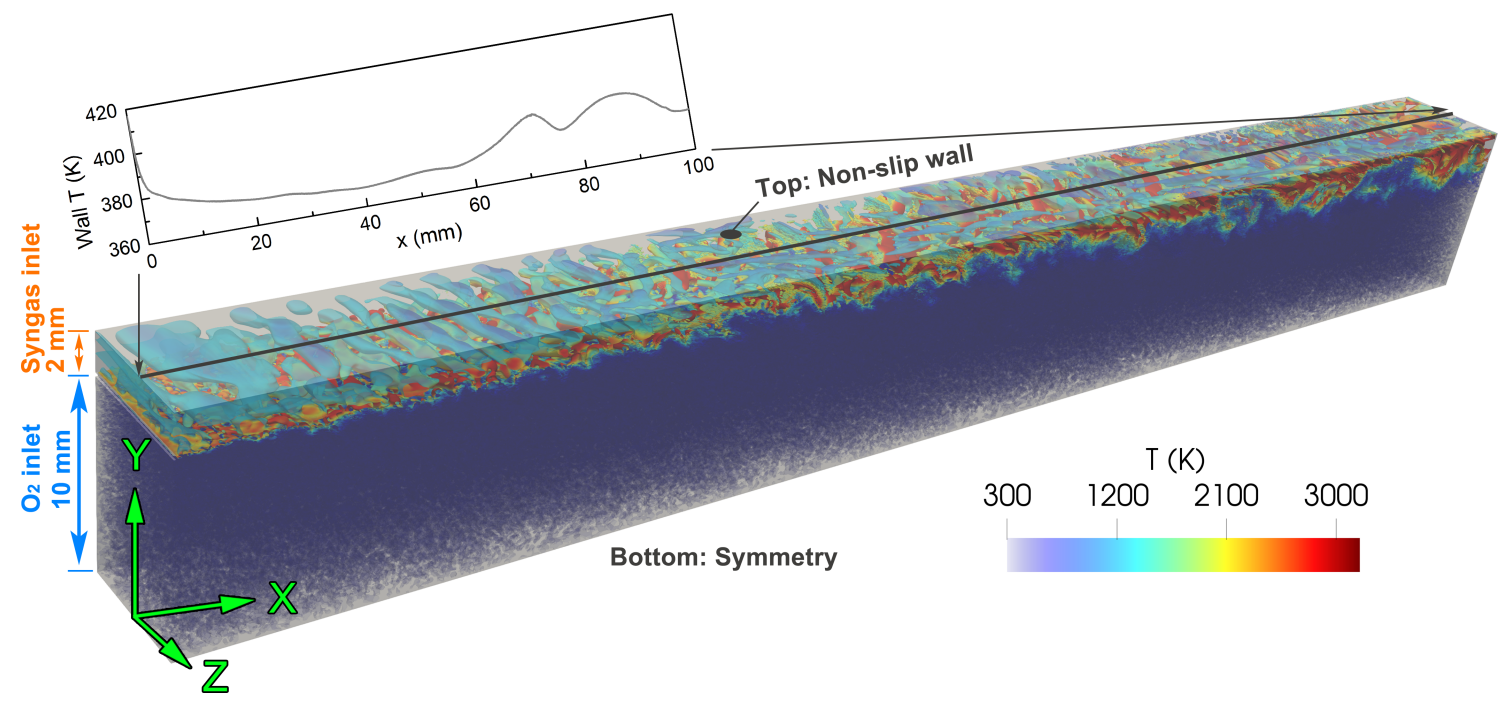

Figure 1: Instantaneous iso-surface of $Q$ criterion [38] predicted by ANN-DNS: $Q=10^{7} \mathrm{~s}^{-2}$, colored by gas temperature. Top graph: distribution of instantaneous wall temperature along the centerline.

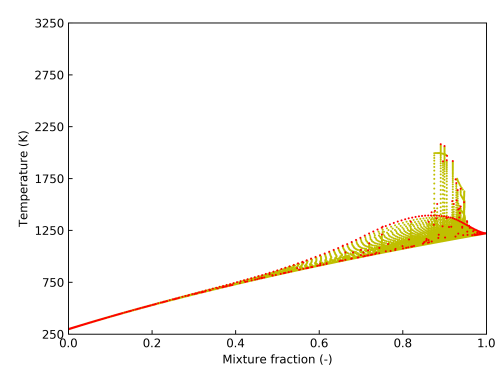

(a) $t=81 \mu \mathrm{s}$

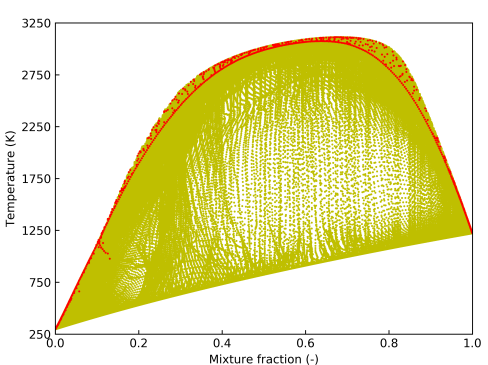

(b) $t=0.3 \mathrm{~ms}$

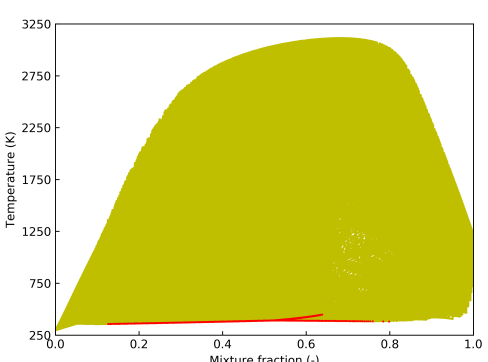

(c) $t=0.9 \mathrm{~ms}$

Figure 2: Scatter plots of instantaneous temperature against mixture fraction of every stochastic particle in the micromixing canonical problem at (a) $t=81 \mu \mathrm{s}$, (b) $t=0.3 \mathrm{~ms}$, and (c) $t=0.9 \mathrm{~ms}$. Red: the 1000 stochastic particles at the current time $t$. Yellow: Time history of the 1000 stochastic particles over the duration $0-t$.

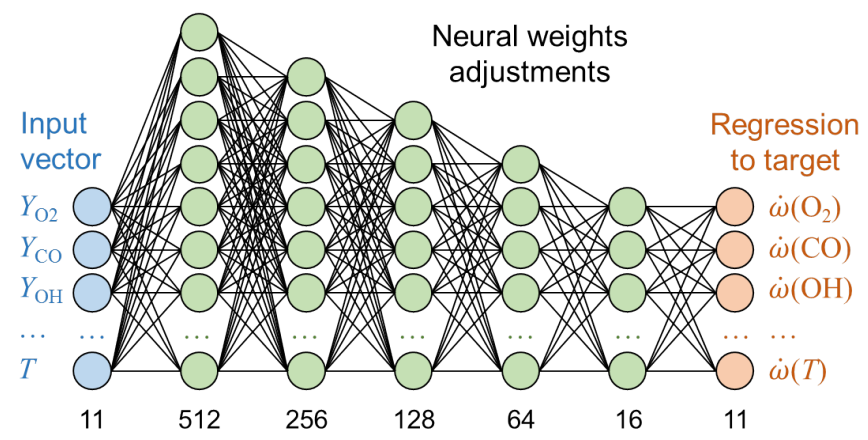

Figure 3: Sketch of the ANN training process from the micro-mixing database. 


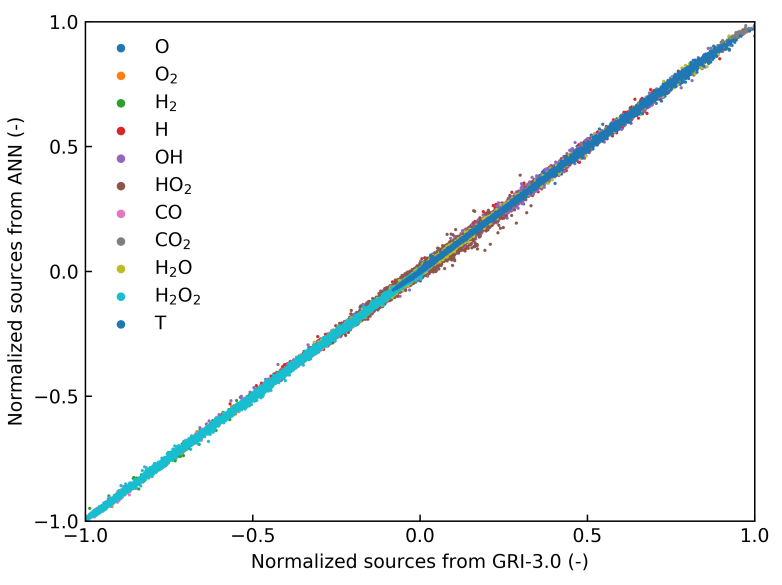

(a) Main ANN

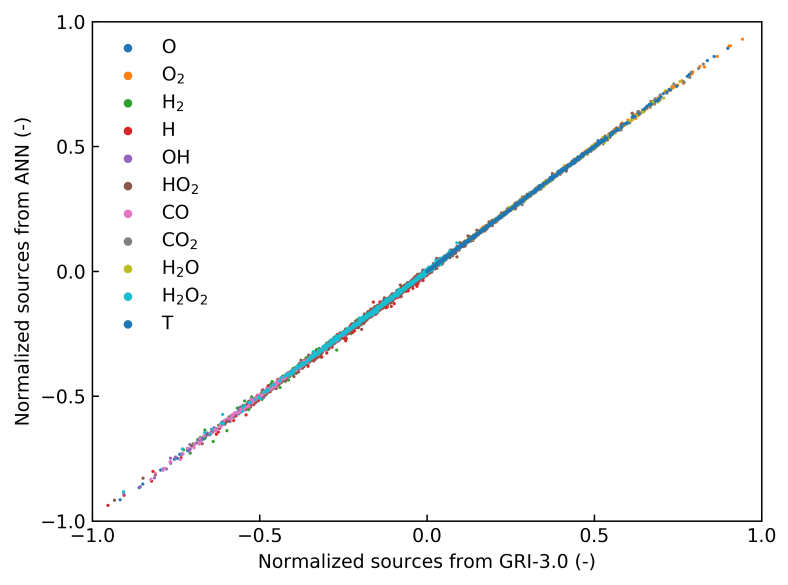

(b) Second ANN, $Y_{\mathrm{O}_{2}}>0.9$

Figure 4: Comparison of the ANNs prediction on normalized sources of the 10 species and temperature against the GRI-3.0 target values for the testing database unseen during training.
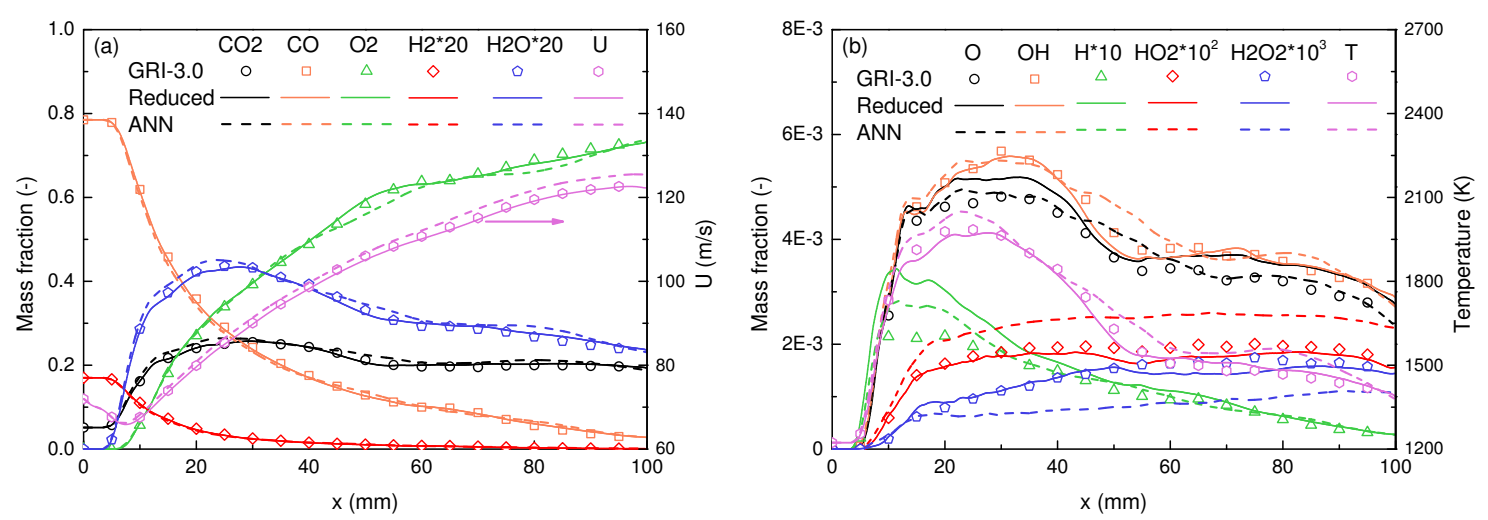

Figure 5: Averaged distributions along the planar fuel-jet centerline $(\mathrm{y}=11 \mathrm{~mm})$. Symbols: GRI-3.0. Solid line: Reduced mechanism (Table 2). Dashed line: ANN chemistry. (a) Streamwise velocity and major species mass fractions: $\mathrm{CO}_{2}, \mathrm{CO}, \mathrm{O}_{2}, \mathrm{H}_{2}$ and $\mathrm{H}_{2} \mathrm{O}$. (b) Temperature and radicals and minor species mass fractions: $\mathrm{O}, \mathrm{OH}, \mathrm{H}$, $\mathrm{HO}_{2}$, and $\mathrm{H}_{2} \mathrm{O}_{2}$. 2D case. 

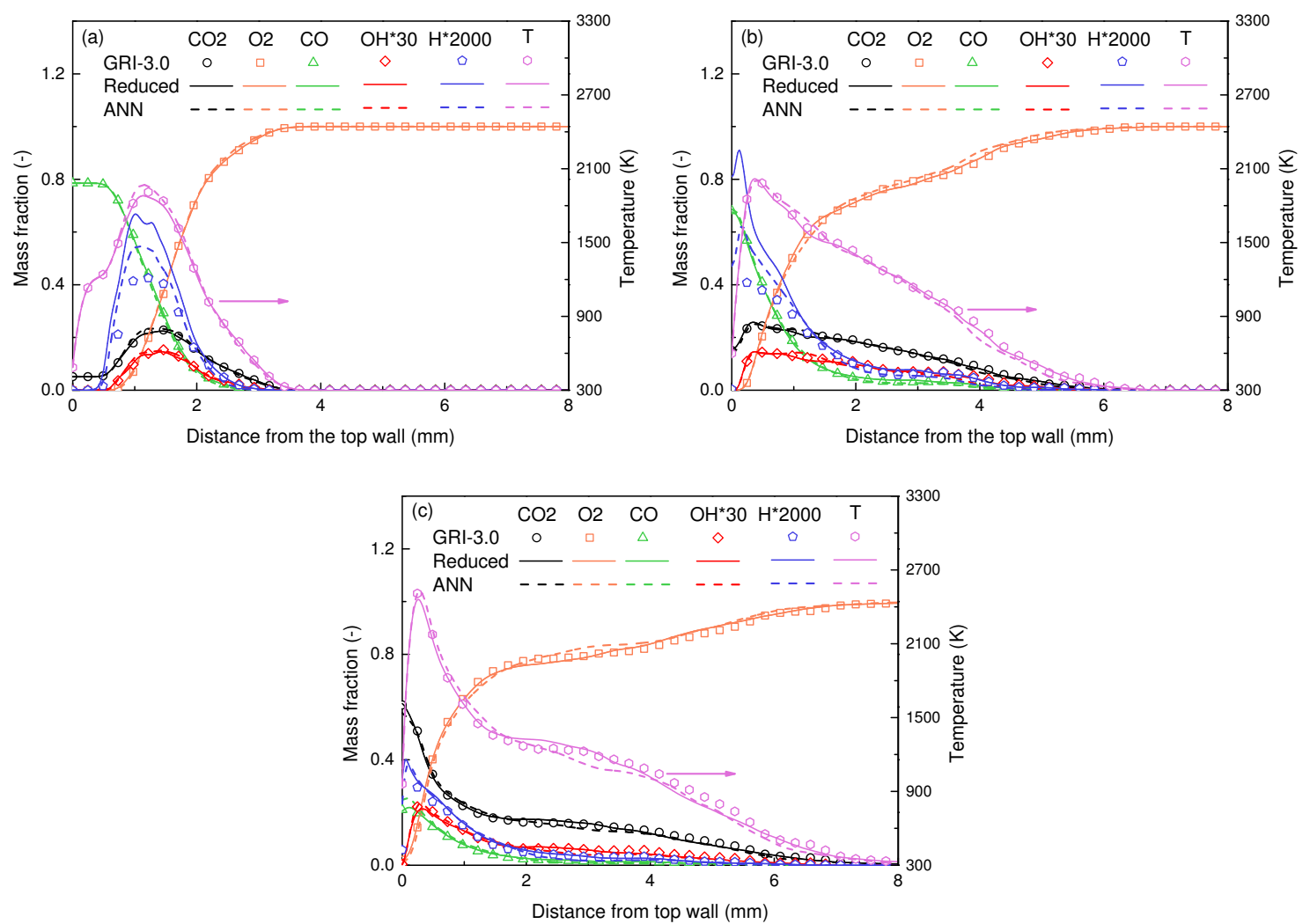

Figure 6: Spanwise averaged distributions of $\mathrm{CO}_{2}, \mathrm{O}_{2}, \mathrm{CO}, \mathrm{OH}, \mathrm{H}$ mass fractions and temperature. Symbols: GRI3.0. Solid line: Reduced mechanism (Table 2). Dashed line: ANN chemistry. (a): $15 \mathrm{~mm}$ from the nozzle inlet. (b): $50 \mathrm{~mm}$ from the nozzle inlet. (c): $80 \mathrm{~mm}$ from the nozzle inlet. 2D case.
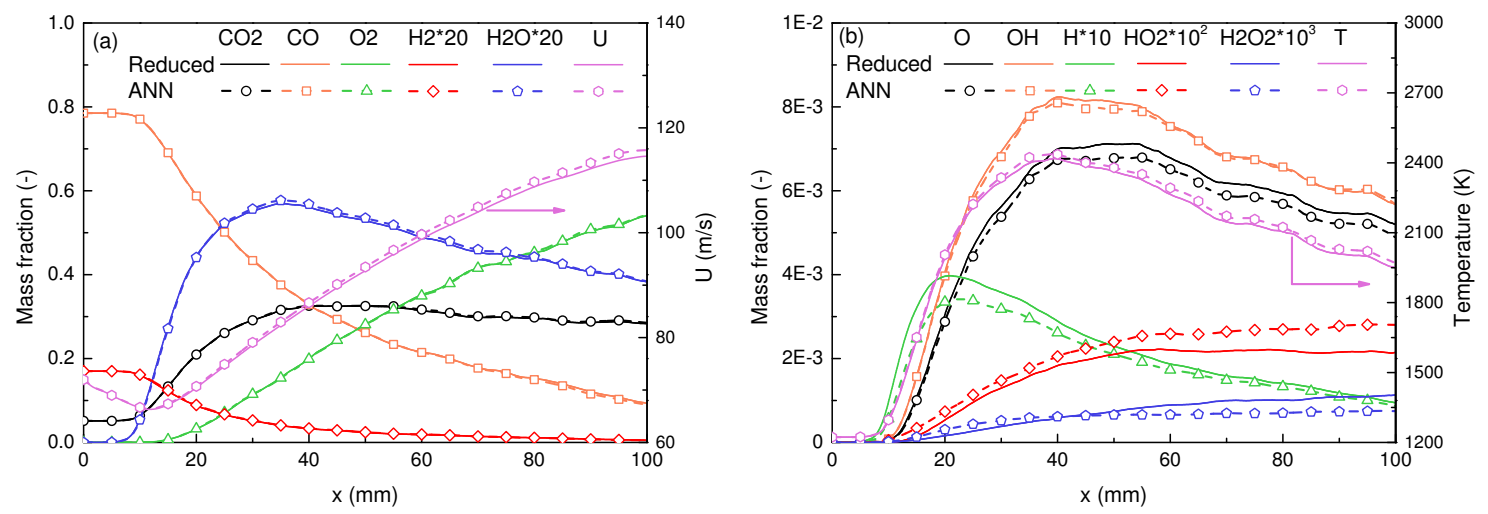

Figure 7: Averaged distributions along the planar fuel-jet centerline $(\mathrm{y}=11 \mathrm{~mm})$. Solid line: Reduced mechanism (Table 2). Dashed line with symbols: ANN chemistry. (a) Streamwise velocity and major species mass fractions: $\mathrm{CO}_{2}, \mathrm{CO}, \mathrm{O}_{2}, \mathrm{H}_{2}$ and $\mathrm{H}_{2} \mathrm{O}$. (b) Temperature and radicals and minor species mass fractions: $\mathrm{O}, \mathrm{OH}, \mathrm{H}, \mathrm{HO}_{2}$, and $\mathrm{H}_{2} \mathrm{O}_{2}$. 3D case. 

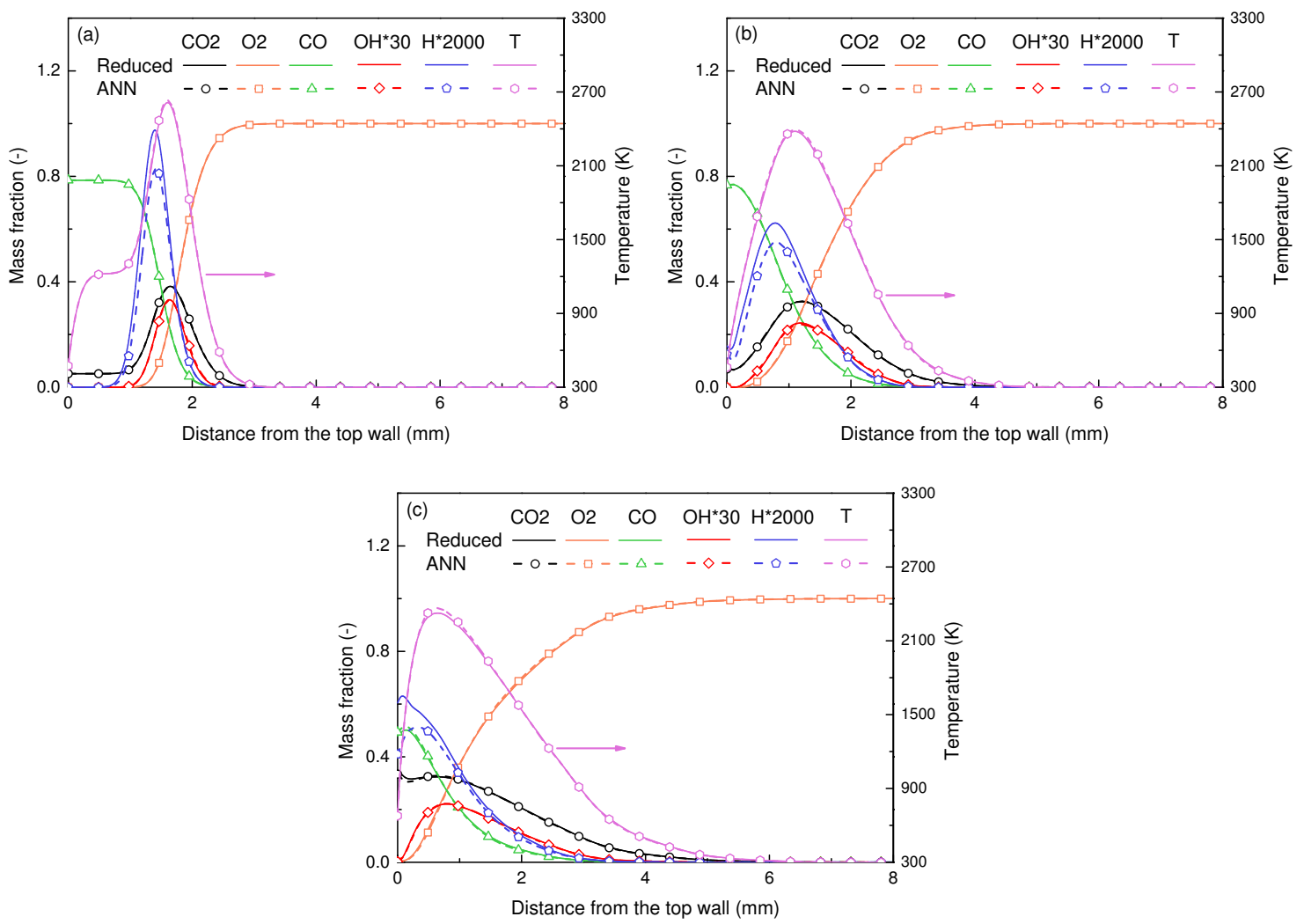

Figure 8: Spanwise averaged distributions of $\mathrm{CO}_{2}, \mathrm{O}_{2}, \mathrm{CO}, \mathrm{OH}, \mathrm{H}$ mass fractions and temperature. Solid line: Reduced mechanism (Table 22). Dashed line with symbols: ANN chemistry. (a) $15 \mathrm{~mm}$ from the nozzle inlet. (b) 50 $\mathrm{mm}$ from the nozzle inlet. (c) $80 \mathrm{~mm}$ from the nozzle inlet. 3D case.

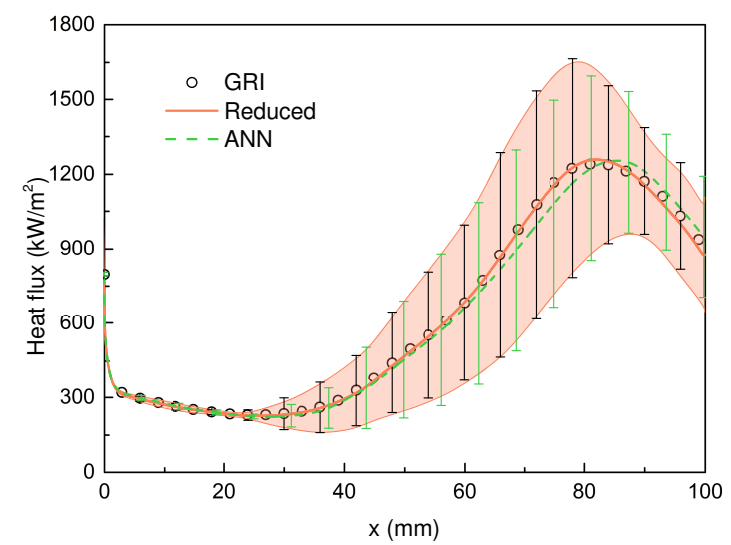

(a) $2 \mathrm{D}$ case

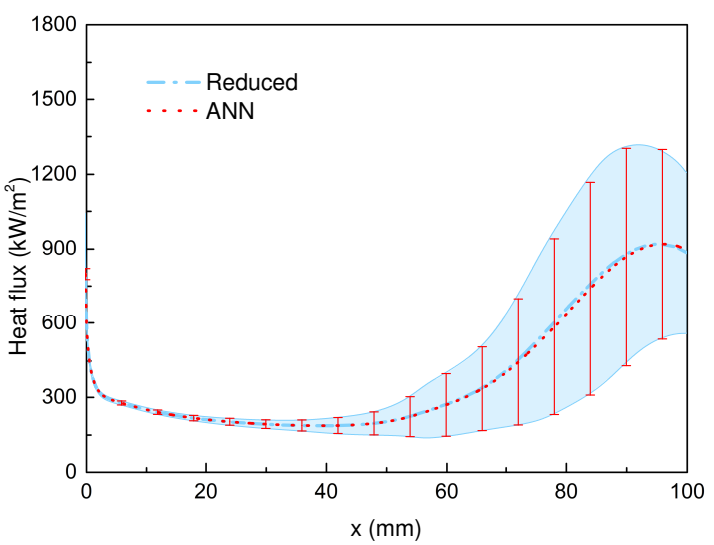

(b) $3 \mathrm{D}$ case

Figure 9: Averaged distribution of wall heat flux at the upper wall. Mean \pm RMS. 2D (a) Symbols: GRI-3.0. Solid line and orange: Reduced mechanism (Table 2). Dashed line and green: ANN chemistry. 3D (b) Dash dotted line and blue: Reduced mechanism (Table 22). Dotted line and red: ANN chemistry. 


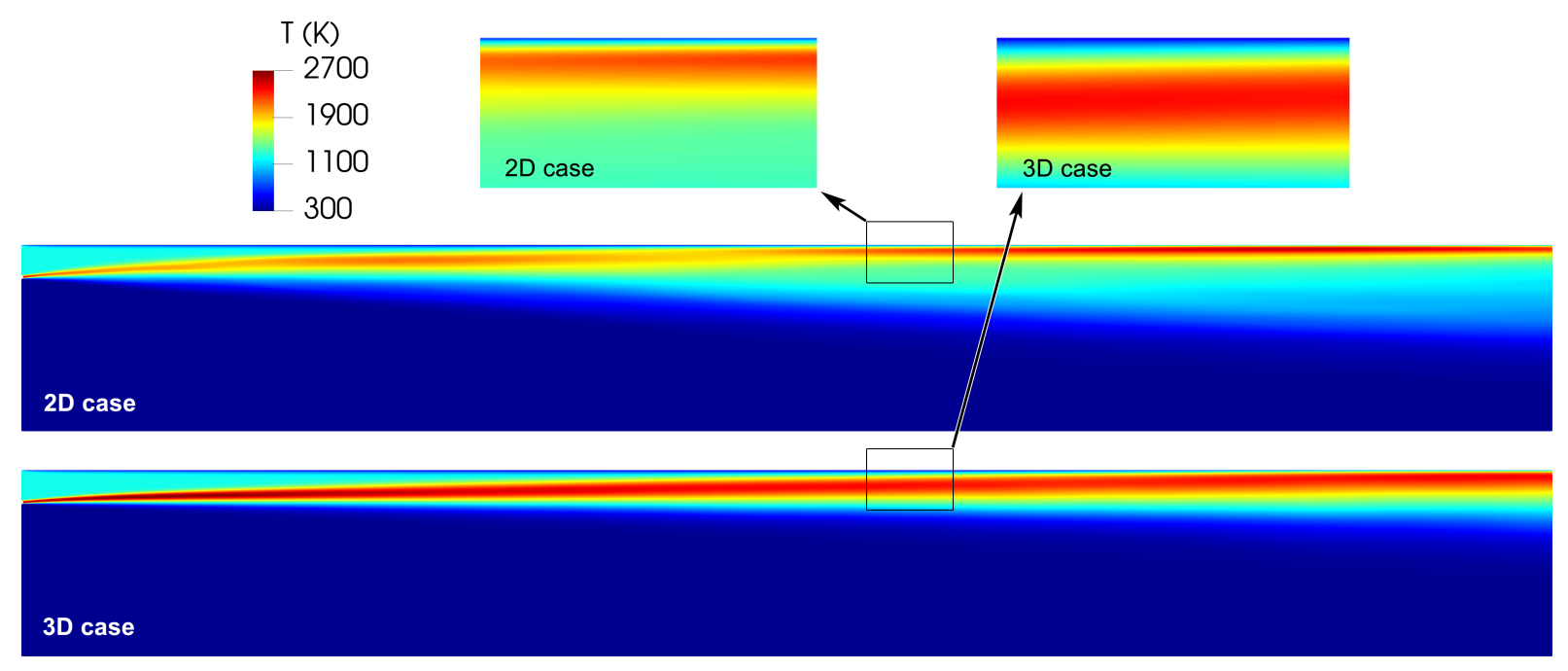

Figure 10: Averaged distribution of temperature predicted by ANN chemistry in 2D and 3D cases. The zoomed-in sub-figures show the thermal boundary layers near the upper wall.

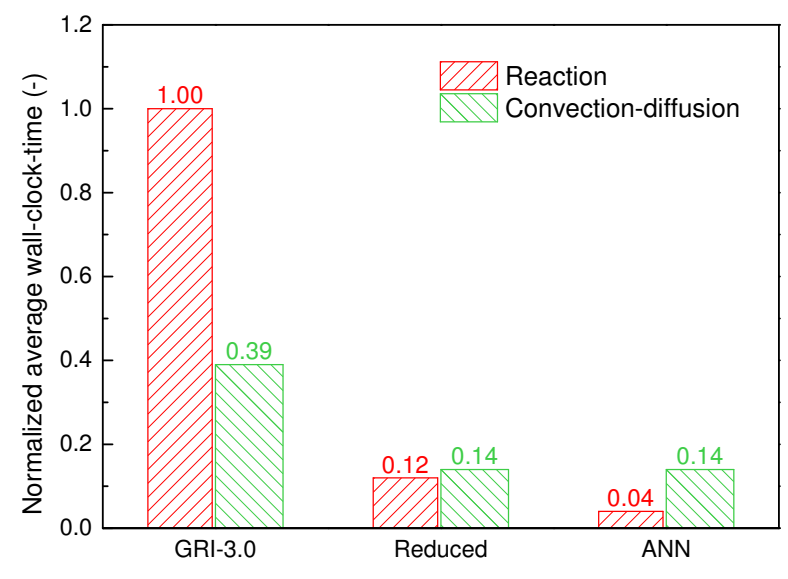

Figure 11: Normalized average wall-clock-time per time step for solving reaction sources and the convective-diffusive part of the equations in 2D DNS coupled with GRI-3.0, 11-species reduced mechanism (Table 2), and ANN. (Normalization by GRI-3.0.) 


\section{List of Supplementary materials}

$\mathrm{S} 1$. The Chemkin file of the 11-species reduced mechanism for syngas oxy-flame.

S2. Instructions of coupling the ANNs with a flow solver.

S3. The main ANN in TensorFlow format, ready for coupling.

S4. The second ANN in TensorFlow format, ready for coupling. 